\title{
Evolution of Cavitary Lesion in a Case of COVID-19 Pneumonia: A Rare Entity
}

\author{
Debasis Behera ${ }^{1}$ Saswat Subhankar ${ }^{1} \quad$ Rajesh Venkataram $^{2} \quad$ Suman Kumar Jagaty ${ }^{1} \quad$ C. M. Rao ${ }^{1}$ \\ Ruchi Rekha Behera ${ }^{3}$ Pragyan Rout ${ }^{1}$ \\ ${ }^{1}$ Department of Pulmonary, Critical Care and Sleep Medicine, KIMS, \\ Bhubaneswar, Odisha, India \\ ${ }^{2}$ Department of Pulmonary Medicine, KS Hegde Medical Academy, \\ Mangalore, Karnataka, India \\ ${ }^{3}$ Department of Paediatrics, SCB Medical College, Cuttack, Odisha, \\ India \\ J Health Allied Sci Nu 2022;12:83-86.

\begin{abstract}
Address for correspondence Debasis Behera, MD, Department of Pulmonary, Critical Care and Sleep Medicine, KIMS, Bhubaneswar,
\end{abstract} \\ Odisha 751024, India (e-mail: drdebasis8@gmail.com).
}

Introduction Toward the end of 2019, a novel coronavirus was identified as the causative organism of a cluster of pneumonia cases in Wuhan, China, spreading rapidly resulting in an epidemic throughout China, followed by a global pandemic. The initial radiological findings of coronavirus disease 2019 (COVID-19) pneumonia at the early stage on a computed tomography (CT) scan include multiple small patchy shadows and interstitial inflammation, predominantly distributed in the peripheral one-third of the lungs. Gradually, it develops into multiple ground glass opacities and infiltrates in the lungs. Furthermore, pulmonary consolidation is observed, but pleural effusion is rare.

Objective From time of presentation to complete recovery, CT scans show significant morphological changes in the lesions, but very few literatures have reported cavitary lesion in the lungs. We present the case of a 64-year-old patient with COVID-19 pneumonia who had typical manifestations of the disease on a CT scan along with constantly changing small cavity in the lung.

Discussion Delayed cavitation is a rarely described radiological manifestation of COVID-19. Atypical presentations of COVID-19 raise possibility of other infections or additional diagnoses. Bacterial and fungal infection should be excluded, as well as cavity-causing organisms. Although the proper mechanism of cavitation in COVID-19 pneumonia is not known, it may be due to intra- alveolar hemorrhage, diffuse alveolar damage, and necrosis of parenchymal cells based on findings of autopsy reports. Early and late complications associated with COVID-19 are yet to be defined. Conclusion Common causes of cavitary lesions must be investigated properly in all patients. Most cases are self-limited hence managed conservatively. The clinical spectrum of disease due to COVID-19 continues to evolve. Physicians must be aware of evolving radiological findings of COVID-19 and must conduct regular follow-up of convalescent patients with COVID-19 to ensure complete recovery. published online

August 20, 2021
DOI https://doi.org/

$10.1055 / \mathrm{s}-0041-1734401$ ISSN 2582-4287 (c) 2021. Nitte (Deemed to be University).

This is an open access article published by Thieme under the terms of the Creative Commons Attribution-NonDerivative-NonCommercial-License, permitting copying and reproduction so long as the original work is given appropriate credit. Contents may not be used for commercial purposes, or adapted, remixed, transformed or built upon. (https://creativecommons.org/licenses/by-nc-nd/4.0/). Thieme Medical and Scientific Publishers Pvt. Ltd. A-12, 2nd Floor, Sector 2, Noida-201301 UP, India 


\section{Introduction}

Toward the end of 2019, a novel coronavirus was identified as the causative organism of a cluster of pneumonia cases in Wuhan, China. It rapidly spread, resulting in an epidemic throughout China, followed by a global pandemic. ${ }^{1}$ The initial radiological findings of coronavirus disease 2019 (COVID-19) pneumonia at the early stage on a computed tomography $(\mathrm{CT})$ scan include multiple small patchy shadows and interstitial inflammation, predominantly distributed in the peripheral one-third of the lungs. Gradually, it develops into multiple ground glass opacities and infiltrates in the lungs. Furthermore, pulmonary consolidation is observed, but pleural effusion is rare. ${ }^{2}$ From the time of presentation to complete recovery, CT scans show significant morphological changes in the lesions, but very few literatures have reported cavitary lesion in the lungs. We present the case of a 64-year-old patient with COVID-19 pneumonia who had typical manifestations of the disease on a CT scan along with constantly changing small cavity in the lung.

\section{Case Report}

A 71-year-old male patient presented to our hospital complaining of fever, cough, fatigue, myalgia for 1 week, and shortness of breath for 2 days. He had no previous history of hypertension, diabetes, coronary heart disease, and tuberculosis. The first CT scan showed fibrobronchiectatic changes in apical and posterior segments of right upper lobe, patchy round ill-defined multiple ground glass opacities bilaterally that was amounting to CT score of 5/25 ( - Fig. 1). A laboratory examination showed white blood cell (WBC) of $18.7 \mathrm{x}$ $10^{9}$ cells/L, the lymphocyte count was $2.89 \times 10^{9}$ cells/L, the C-reactive protein (CRP) level was $227 \mathrm{U} / \mathrm{mL}$, serum ferritin was $214 \mu \mathrm{g} / \mathrm{L}$, lactate dehydrogenase (LDH) was $546 \mathrm{U} / \mathrm{L}$, and D-dimer was $0.43 \mu \mathrm{g} / \mathrm{mL}$. Reverse transcription-polymerase chain reaction test for COVID-19 was positive on a pharyngeal swab, confirming the diagnosis of COVID-19 pneumonia. Initially he was managed in isolation ward because of his

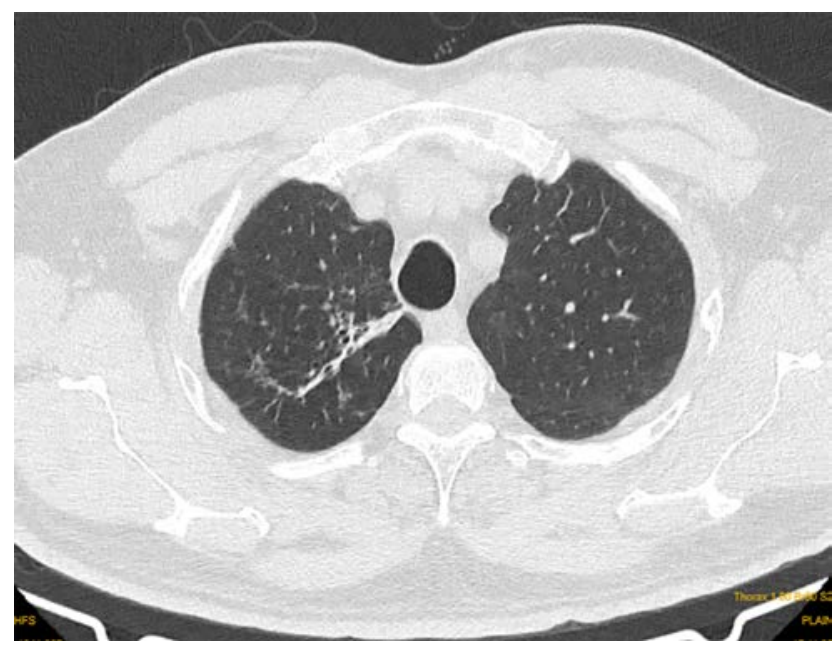

Fig. 1 CT scan at the time of admission showing fibrobronchiectatic changes in apical and posterior segments of right upper lobe, patchy round ill-defined multiple ground glass opacities bilaterally. stable vitals, but gradually his condition was deteriorated, and oxygen requirement was increased for which he was shifted to intensive care unit for further management. A repeat chest CT scan showed that the ground glass opacities area as well as areas of consolidation involving all the areas of both lungs became diffuse; some of them show septal thickening (crazy pavement) CT score 21/25 (-Fig. 2). The laboratory results showed marginally raised WBC with gross increase in the CRP, D-dimer, ferritin, and LDH level because of worsening COVID-19 status. He was managed with intravenous antibiotics, Remdesivir, steroid, anticoagulant, and other supportive measures. Respiratory support was given with nonrebreathing mask and intermittent noninvasive ventilation. Because of increasing oxygen requirement with worsening inflammatory markers, tocilizumab was considered for impending cytokine storm. Two-dimensional echocardiogram and computed tomography pulmonary angiogram (CTPA) was done to rule out any cardiovascular etiology because of persistent shortness of breath but both did not reveal any abnormal findings. However, in CTPA parenchymal window there was pneumothorax in right side and a cavitary lesion of size $9 \times 10 \times 9.5 \mathrm{~mm}$ in anterior segment of right upper lobe ( $\boldsymbol{- \text { Fig. }} \mathbf{3}$ ) for which intercostal tube drainage was inserted that was removed after 4 days as lung was expanded after confirming in CT thorax. Gradually

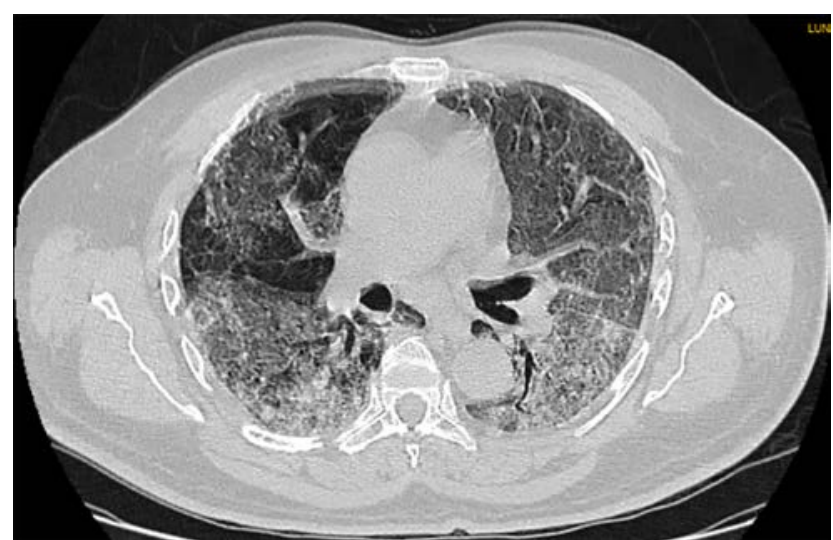

Fig. 2 CT scan showing ground glass opacities as well as consolidation involving all the areas of both lungs; some of them showing septal thickening (crazy pavement pattern).

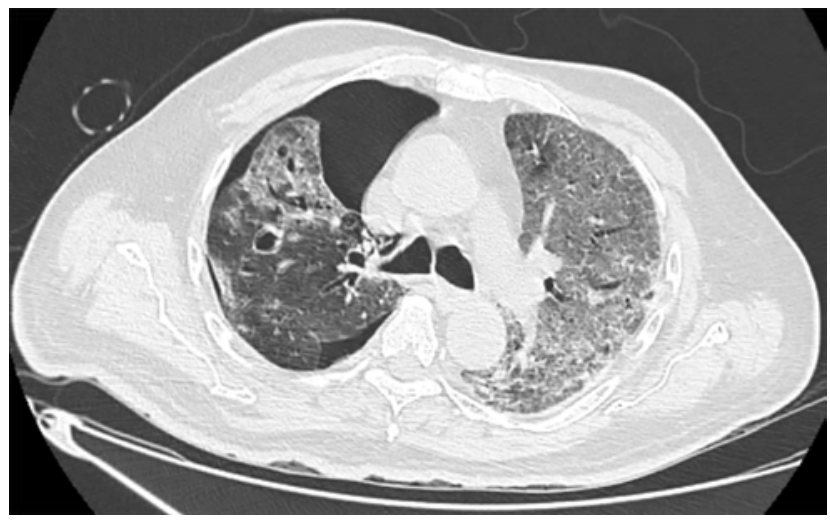

Fig. 3 CT scan showing pneumothorax in right side and a cavitary lesion of size $9 \times 10 \times 9.5 \mathrm{~mm}$ in anterior segment of right upper lobe. 


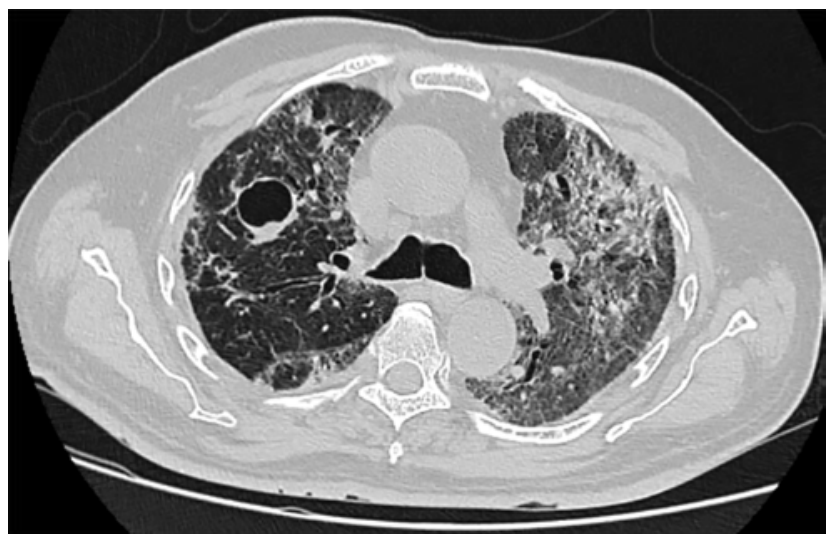

Fig. 4 CT scan at the time of discharge showing a cavitary lesion of size $2.3 \times 2.5 \times 2 \mathrm{~cm}$ that was increased in size compared with the previous $\mathrm{CT}$ scan along with fibrotic bands and traction bronchiectasis.

his condition was improving but in view of a new cavitary lesion bronchoscopic guided lavage was performed and was negative for acid fast bacillus, bacterial, and fungal infection. Serological markers for fungal infection like galactomannan and $\beta-1,3-D$-glucan were also negative. Gradually patient condition improved, and his oxygen requirement came down with inflammatory markers around normal limits. CT thorax at the time of discharge was showing a cavitary lesion of size $2.3 \times 2.5 \times 2 \mathrm{~cm}$ that was increased in size compared with the previous $\mathrm{CT}$ thorax along with fibrotic bands and traction bronchiectasis ( - Fig. 4). Hence, patient was discharged with antifibrotics, newer oral anticoagulants, and low-dose steroids with advice of regular follow-up.

\section{Discussion}

COVID-19 is a pulmonary disease caused by severe acute respiratory syndrome coronavirus 2 (SARS-CoV-2). This virus can grow in alveolar epithelial cells or bronchiole mucosa, resulting in mixed injury of pulmonary parenchyma and interlobular septa, interstitial tissue of alveoli, and periacinar microvascular network., ${ }^{3,4}$ For most recovered viral pneumonia, the lung lesions are generally absorbed and dissipated, but for COVID-19, pneumonia image change in convalescence period needs further observation. In this case, the patient was healthy and had no smoking history. During the course of the disease, no bacteria were detected in the blood and sputum culture, and also the results of WBC count and procalcitonin did not support bacterial infection. In this patient, pulmonary cavity appeared on day 22 . As a result, the pulmonary cavity of this patient was considered to be caused by SARS-CoV-2 infection, possibly leading to a large area of alveolar and surrounding pulmonary interstitial destruction and fusion.

The early manifestations of COVID-19 pneumonia on a CT scan are multiple ground glass opacities peripherally in both lungs. With the development of the disease, the lesions expand with increase in consolidation. After effective treatment, the number of lesions decrease, and the ground glass opacities absorb. Some lesions transform into linear opacities in a short period. However, there are very few literatures reporting small cavities in the lungs during the resolution phase. ${ }^{5,6}$

In this case, the initial findings on the CT scan were multiple ground glass opacities and linear opacities distributed in the peripheral third of the lungs, which are the characteristics pulmonary imaging manifestations of COVID-19 pneumonia. Repeat CT scan showing that the ground glass opacities had been reduced by resolution and few linear opacities remained. The unique manifestation in this case was the small cavity in right lung during the resolution phase that was gradually increased in size. The small cavity in the lung of this patient during the resolution period might be explained by the focal hemorrhage and necrosis of the lung tissue forming this small cavity after drainage through bronchioles; the congested blood vessels of the alveolar septum around the cavity and the edema around the cavity formed the thin wall. ${ }^{7}$

The small cavities might also be explained by the fact that the bronchioles in the lungs were blocked by mucus and mucus plugs, the overinflation of the alveoli, the rupturing of the alveolar septum, and the subsequent formation of small cysts, which appear as small cavities on the chest CT. The cavity consolidated and then shrank, eventually being absorbed or disappearing.

Delayed cavitation is rarely described as a radiological manifestation of COVID-19. Atypical presentations of COVID-19 raise possibility of other infections or additional diagnoses. Bacterial and fungal infection should be excluded, as well as cavity-causing organisms. Although the proper mechanism of cavitation in COVID-19 pneumonia is not known, it may be due to intraalveolar hemorrhage, diffuse alveolar damage, and necrosis of parenchymal cells based on findings of autopsy reports. ${ }^{8,9}$

\section{Conclusion}

Common causes of cavitary lesions must be investigated properly in all patients. Most cases are self-limited hence managed conservatively. The clinical spectrum of disease due to COVID-19 continues to evolve. Early and late complications associated with COVID-19 are yet to be defined. Physicians must be aware of evolving radiological findings of COVID-19 and must conduct regular follow-up of convalescent patients with COVID-19 to ensure complete recovery.

\section{Authors' Contributions}

All the authors contributed substantially to the conception or design of the work, the acquisition, analysis, or interpretation of data for the work; drafting of the work or critical revision of important intellectual content; final approval of the version to be published; and agreement to be accountable for all aspects of the work in ensuring that questions related to the accuracy or integrity of any part of the work are appropriately investigated and resolved.

\section{Conflict of Interest}

None declared. 


\section{References}

1 World Health Organization. Novel coronavirus-China[EB/OL]. (2020-01-12) [2020-02-10]. Available at: https://www.who. int/csr/don/12-january-2020-novel-coronavirus-china/en. Accessed July 17, 2021

2 Pan F, Ye T, Sun P, et al. Time course of lung changes at chest CT during recovery from coronavirus disease 2019 (COVID-19. Radiology 2020;295(3):715-721

3 Liu K, Fang YY, Deng Y, et al. Clinical characteristics of novel coronavirus cases in tertiary hospitals in Hubei Province. Chin Med J (Engl 2020;133(9):1025-1031

4 Pan Y, Guan H, Zhou S, et al. Initial CT findings and temporal changes in patients with the novel coronavirus pneumonia (2019-nCoV): a study of 63 patients in Wuhan, China. Eur Radiol 2020;30(6):3306-3309

5 Guan HX, Xiong Y, Shen NX, et al. Clinical and thin-section CT features of patients with 2019-nCoV-pneumonia. Radiol Pract 2020. doi: 10.13609/ j.cnki.1000- 0313.2020.02.001
6 Shi $\mathrm{H}$, Han $\mathrm{X}$, Jiang $\mathrm{N}$, et al. Radiological findings from 81 patients with COVID-19 pneumonia in Wuhan, China: a descriptive study. Lancet Infect Dis 2020;20(4):425-434

$7 \mathrm{Xu} \mathrm{Z}$, Shi L, Wang Y, et al. Pathological findings of COVID-19 associated with acute respiratory distress syndrome. Lancet Respir Med 2020;8(4):420-422

8 Menter T, Haslbauer JD, Nienhold R, et al. Postmortem examination of COVID-19 patients reveals diffuse alveolar damage with severe capillary congestion and variegated findings in lungs and other organs suggesting vascular dysfunction. Histopathology 2020;77(2):198-20910.1111/his.14134 [Epub ahead of print: 04 May 2020]

9 Yao XH, Li TY, He ZC, et al. [A pathological report of three COVID-19 cases by minimal invasive autopsies]. Zhonghua Bing Li Xue Za Zhi 2020;49(5):411-417 